TITLE:

\title{
The effect of electric current on chemical bonding of hydrogen adsorption on an aluminum nanowire
}

\author{
$\operatorname{AUTHOR(S):~}$ \\ Senami, Masato; Matsunaga, Shunji
}

\section{CITATION:}

Senami, Masato ... [et al]. The effect of electric current on chemical bonding of hydrogen adsorption on an aluminum nanowire. International Journal of Quantum Chemistry 2019, 119(22): e26004.

\section{ISSUE DATE:}

2019-11-15

URL:

http://hdl.handle.net/2433/244805

\section{RIGHT:}

This is the peer reviewed version of the following article: International Journal of Quantum Chemistry, 119(22), e26004 which has been published in final form at https://doi.org/10.1002/qua.26004. This article may be used for non-

commercial purposes in accordance with Wiley Terms and Conditions for Use of Self-Archived Versions.; The full-text file will be made open to the public on 10 October 2020 in accordance with publisher's 'Terms and Conditions for SelfArchiving'.: This is not the published version. Please cite only the published version.; この論文は出版社版でありません 。引用の際には出版社版をご確認ご利用ください。 


\title{
The effect of electric current on chemical bonding of hydrogen adsorption on an aluminum nanowire
}

\author{
Masato Senami* and Shunji Matsunaga \\ Department of Micro Engineering, Kyoto University, Kyoto 615-8540, Japan
}

(Dated: July 10, 2019)

\begin{abstract}
The effect of electric current on hydrogen adsorption on an aluminum nanowire surface is studied by using nonequilibrium green function method. We choose the models studied in the previous work of one of the authors as an aluminum nanowire model and hydrogen adsorbed one. These nanowire models have conductive ability, since the aluminum part of these models is metallic. It is confirmed that electric current affects the strength of the adsorption of hydrogen atoms and the change of the bonding of hydrogen to aluminum nanowire surface is larger for larger current. However, the change of the chemical bonding is negligibly small within the bias voltage $\leq 0.5 \mathrm{~V}$.
\end{abstract}

*Electronic address: senami@me.kyoto-u.ac.jp 


\section{INTRODUCTION}

Hydrogen storage material is a key factor for the hydrogen economy. In addition to the high storage capacity, low activation energies of adsorption and desorption of hydrogen atoms are required for the successful material. For these requirements, aluminum nano-structures, which have high surface-to-bulk ratio, are studied in many literature [1-25]. Particularly, aluminum cluster such as $\mathrm{Al}_{13}$ have been studied widely and have been reported to have high weight percent ratio and low or no energy barrier for the adsorption of hydrogen atom or molecule, while the activation energy of the desorption of hydrogen atom is generally high.

In our previous works $[18,23]$, the aluminum nanowire, which has the structure tying $\mathrm{Al}_{13}$ clusters one to the next, was studied as a candidate for hydrogen storage material. The chemical bonding between hydrogen and aluminum atoms was studied and classified as the covalent bond [18]. In the work [23], the potential energy surface of hydrogen adsorption on aluminum nanowire was studied, and the migration path of the hydrogen atom adsorbed on the aluminum nanowire was clarified. The aluminum nanowire has lower surface-tobulk ratio than the aluminum cluster and, however, can be used as a conductor because nanowire structure can be connected to electrodes. We consider that electric current through the aluminum nanowire may affect the chemical bonding between hydrogen and aluminum atoms. Particularly, the activation energy of the hydrogen desorption from aluminum nanostructures may be lowered to a moderate level. Actually, the work [25] reported that large electric current through $\mathrm{AlH}_{3}$ cluster induces to form a chemical bonding between hydrogen atoms. This result highly stimulates our study of the effect of the electric current on the chemical bonding in the aluminum nanowire, though the effect of electrodes was not included and the current density was large in the work.

Hence, in this work, we check this effect in aluminum nanowire with nonequilibrium Green's function (NEGF) method for the implementation of the electric current in numerical computations, while the highest occupied molecular orbital is mixed with a plane wave in the work [25]. NEGF method is not suitable for very large current density as 0.1 [a.u.], and hence the electric current in this work is much smaller than the previous work. However, the magnitude of the current by NEGF is more plausible in practical devices. Therefore, the confirmation with NEGF method is considered to be meaningful, even if computational conditions are different largely. 
This paper is organized as follows. In the next section, local physical quantities defined in Rigged QED are briefly introduced. We can analyze chemical bonding by these quantities and particularly parametrize the strength of chemical bonds by the bond order in Rigged QED. In Sec. III, our computational detail is explained. In Sec. IV, first, we show the results without electric current for comparison. In this work, we used different computational program package from that used in Refs. $[18,23]$. Hence we check the effect of the difference between program codes. Then we show our results of the effect of the electric current on the chemical bonding. The last section is devoted to the summary.

\section{THEORY}

In this paper, we study the effect of electric conduction on the bonding between hydrogen and aluminum atoms in an aluminum nanowire model by local physical quantities based on Rigged QED [26-29]. In Rigged QED, properties of molecules are clarified by these local physical quantities, such as electronic stress tensor density, electronic tension density, kinetic energy density, and the energy density based bond order. Rigged QED provides many tools for the analysis of molecular properties. The surfaces of the atom and molecule are defined with the the kinetic energy density. Covalent and metallic bonds are identified by stress tensor density, ionic bond is identified by the kinetic energy density. The reactivity of molecules can be investigated by the regional electronic chemical potential. In addition, the property of the response of local region in a matter to the electromagnetic field can be analyzed by the local dielectric constant density and the local conductivity. These quantities of Rigged QED are also used in the preceding works [18, 25]. In this section, we explain the formulation of these quantities for electronic structure computations based on quantum mechanics in the nonrelativistic limit.

First, the electronic stress tensor density, $\tau_{e}^{S k l}(\vec{r})$, is formulated as,

$$
\tau_{e}^{S k l}(\vec{r})=\frac{\hbar^{2}}{4 m} \sum_{i} \nu_{i}\left[\psi_{i}^{*}(\vec{r}) \frac{\partial^{2} \psi_{i}(\vec{r})}{\partial x^{k} \partial x^{l}}-\frac{\partial \psi_{i}^{*}(\vec{r})}{\partial x^{k}} \frac{\partial \psi_{i}(\vec{r})}{\partial x^{l}}+\frac{\partial^{2} \psi_{i}^{*}(\vec{r})}{\partial x^{k} \partial x^{l}} \psi_{i}(\vec{r})-\frac{\partial \psi_{i}^{*}(\vec{r})}{\partial x^{l}} \frac{\partial \psi_{i}(\vec{r})}{\partial x^{k}}\right],
$$

where $\hbar$ is the reduced Planck constant, $m$ is the electron mass, $\psi_{i}$ is the $i$-th natural orbital, $\nu_{i}$ is the occupation number of $\psi_{i}$, and $k, l=x, y, z$. It is known that the covalent 
and metallic bonds are identified by the stress tensor density [30, 31]. This tensor $\stackrel{\leftrightarrow}{\tau}_{e}^{S}$ is diagonalized and the eigenvalues and eigenvectors are used for the analysis. The three eigenvalues are denoted as $\tau_{e}^{S 11}(\vec{r}) \leq \tau_{e}^{S 22}(\vec{r}) \leq \tau_{e}^{S 33}(\vec{r})$. For the covalent bond, the largest eigenvalue is positive in a bonding region between two nuclei and the corresponding eigenvector connect two nuclei in a oval shape. This feature is defined as the spindle structure and the covalent bond is identified by this structure [26-29]. The metallic bond can be identified by the feature that all eigenvalues of the stress tensor density are negative and degenerate with each other $[31]$.

The divergence of the stress tensor density is the tension density,

$$
\begin{aligned}
\tau_{e}^{S k}(\vec{r}) & =\partial_{l} \tau_{e}^{S k l}(\vec{r}) \\
& =\frac{\hbar^{2}}{4 m} \sum_{i} \nu_{i}\left[\psi_{i}^{*}(\vec{r}) \frac{\partial \Delta \psi_{i}(\vec{r})}{\partial x^{k}}-\frac{\partial \psi_{i}^{*}(\vec{r})}{\partial x^{k}} \Delta \psi_{i}(\vec{r})+\frac{\partial \Delta \psi_{i}^{*}(\vec{r})}{\partial x^{k}} \psi_{i}(\vec{r})-\Delta \psi_{i}^{*}(\vec{r}) \frac{\partial \psi_{i}(\vec{r})}{\partial x^{k}}\right] .
\end{aligned}
$$

where $\Delta$ is the Laplacian, $\Delta \equiv \Sigma_{j=1}^{3}\left(\partial / \partial x^{j}\right)^{2}$. For a steady state, the tension density is balanced with the Lorentz force density, which is numerically confirmed even in nonequilibrium steady state [32]. Hence, in quantum field theory, a formulation different from phenomenological relaxation time is available for the counter force to the Lorentz force.

The kinetic energy density, $n_{T_{e}}(\vec{r})$, is defined as

$$
n_{T_{e}}(\vec{r})=-\frac{\hbar^{2}}{4 m} \sum_{i} \nu_{i}\left[\psi_{i}^{*}(\vec{r}) \Delta \psi_{i}(\vec{r})+\Delta \psi_{i}^{*}(\vec{r}) \psi_{i}(\vec{r})\right] .
$$

In the definition of this energy density, negative value of kinetic energy density is allowed. Positive kinetic energy regions are consistent with the classical picture of electron movement, while electrons travel in negative kinetic energy regions only by quantum effects. Interface surface, $n_{T_{e}}(\vec{r})=0$, is proposed as the definition of the surface of atoms and molecules [33]. For the ionic bonding, cations and anions are found to be confined in different electronic surfaces [34].

Lastly, the definition of the energy density based bond order is introduced [30, 35]. Two bond orders have been proposed. The first one is based on the energy density at the Lagrange point, while the second one is defined by the regional electronic chemical potential at the point. The Lagrange point, $\vec{r}_{L}$, is the point where the tension density is zero, $\tau_{e}^{S k}\left(\vec{r}_{L}\right)=0$. 
In our work, we use the former one. The bond order based on the energy density between atoms, $\mathrm{A}$ and $\mathrm{B}$, is given as the ratio of the energy density at $\vec{r}_{L}$ to that of the $\mathrm{H}-\mathrm{H}$ bond in a hydrogen molecule,

$$
b_{\epsilon}=\frac{\epsilon_{\tau \mathrm{AB}}^{S}\left(\vec{r}_{L}\right)}{\epsilon_{\tau \mathrm{HH}}^{S}\left(\vec{r}_{L}\right)}
$$

The energy density, $\epsilon_{\tau}^{S}$, is defined as half of the trace of the stress tensor density,

$$
\epsilon_{\tau}^{S}(\vec{r})=\frac{1}{2} \sum_{k=1}^{3} \tau^{S k k}(\vec{r})
$$

In our work, the effect of electric conduction on chemical bonding is studied. The electric current density is known to be defined as

$$
\vec{J}(\vec{r})=\frac{Z_{e} e}{2 m} \sum_{i}\left[-i \hbar \psi_{i}^{\dagger}(\vec{r}) \vec{\nabla} \psi_{i}(\vec{r})-\frac{Z_{e} e}{c} \psi_{i}^{\dagger}(\vec{r}) \vec{A}(\vec{r}) \psi_{i}(\vec{r})+h . c .\right],
$$

where $e$ is the elementary charge, $Z_{e}=-1$, and $\vec{A}(\vec{r})$ is the vector potential.

\section{COMPUTATIONAL DETAILS}

The structures of our nanowire models are based on previous works [18, 23]. One model is a pristine aluminum nanowire model and its structure is shown in Fig. 1. This structure was optimized with keeping the fivefold symmetry and the stability of this model was confirmed in Ref. [15]. This model has the structure of the repetition of $\mathrm{Al}_{13}$ cluster and this nanowire consists of pentagons and one-dimensional atomic chain. The chain is put on the center points of pentagons, and the distance between the center of a pentagon and an aluminum nucleus on the pentagon is $2.48 \AA$. Atoms on the chain are intercalated between pentagons, and the distance of nuclei on the chain from nearby pentagons is 1.34 $\AA$. The second model is hydrogen adsorbed aluminum nanowire. The structure of aluminum nanowire is modified by the adsorption of hydrogen atoms, and the model of hydrogen adsorbed aluminum nanowire is shown in Fig. 2. This structure was optimized with keeping the fivefold symmetry in Ref. [18]. The distance between the center of a pentagon and an aluminum nucleus on the pentagon is reduced to $2.35 \AA$. The internuclear length between hydrogen and aluminum nuclei is $1.57 \AA$.

Electronic structure computations of these models are carried out by OpenMX program package [36]. Computations of OpenMX are based on density functional theory (DFT). 


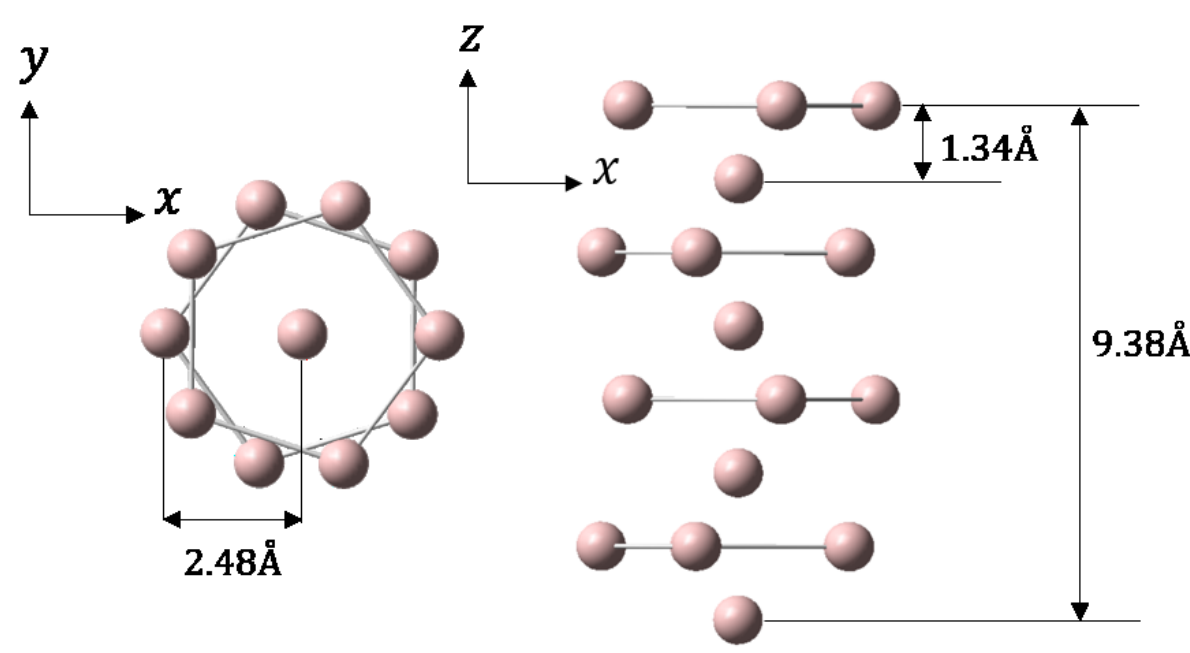

FIG. 1: The structure of pristine aluminum nanowire model. This structure is based on Ref. [18, 23].
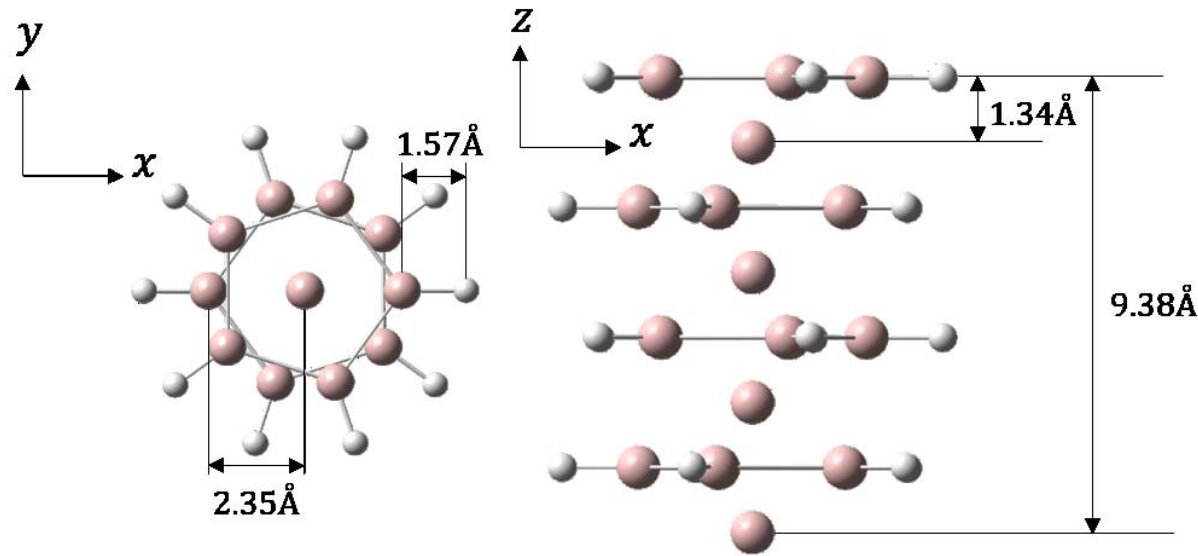

FIG. 2: The structure of hydrogen adsorbed aluminum nanowire model. This structure is based on Ref. $[18,23]$.

Generalized gradient approximation of Perdew-Burke-Ernzerhof (GGA-PBE) is adopted as functionals for correlation interaction. Basis sets are chosen to be Al7.0-s2p2d1 and H5.0-s1 [37]. The size of supercell of our computations is $15 \AA \times 15 \AA \times 10.72 \AA$. Periodic boundary conditions are imposed in our computations, and the size of the supercell, $15 \AA$, is chosen so that effects by next nanowire are negligible. Cutoff energy is set to be 200 Ry.

For computations of conduction states, we also use OpenMX with NEGF method. The same basis set and cutoff energy are adopted. The supercell of these computations are twice the length of Fig. 2 as shown in Fig. 3. The direction of conduction is assumed to be the $z$-direction, and periodic boundary conditions are set for $x$ and $y$ directions. For $z$-direction, 


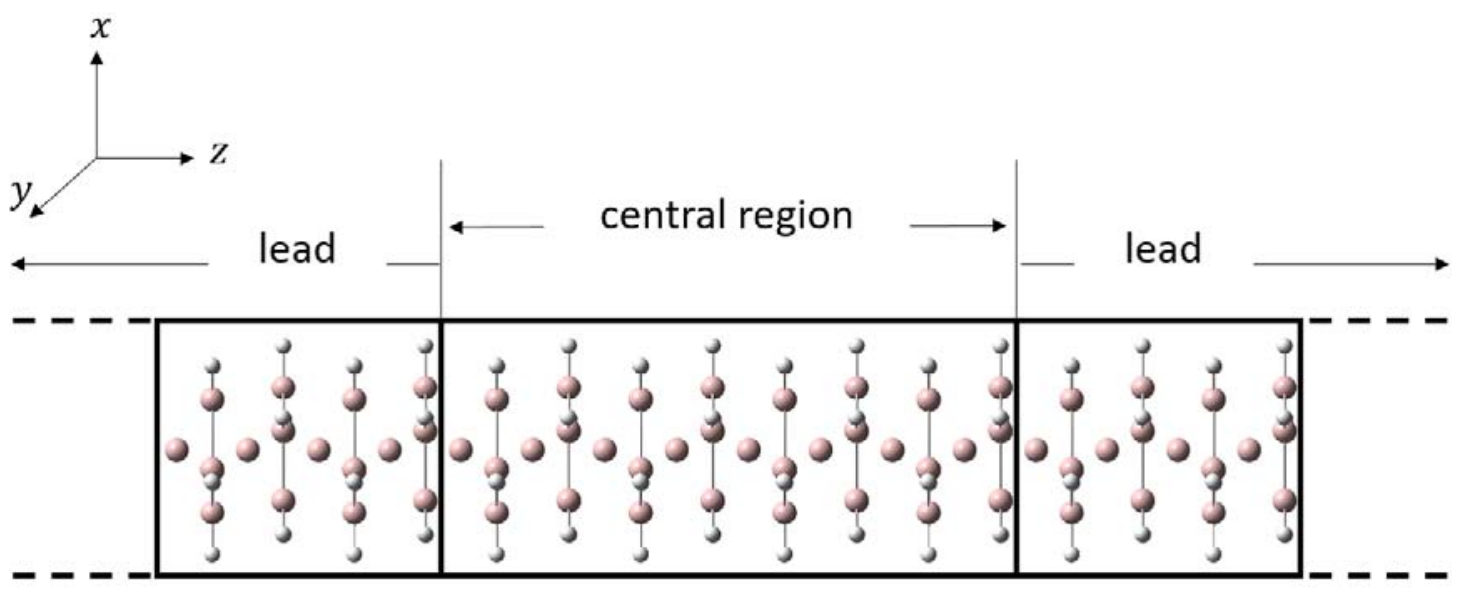

FIG. 3: The hydrogen adsorbed aluminum nanowire model for computations with electric current.

eight pentagon layers are included as electrode parts (left and right leads). The bias voltage is taken as $0.0,0.3$, and $0.5 \mathrm{~V}$ for the check of the dependence on the bias voltage.

After electronic structure computations, local physical quantities based on Rigged QED are computed with these electronic structures. These computations are carried out by QEDynamics program package [38]. Contour plots are depicted in some figures in the next section. A Gaussian filter is used for the purpose of removing some noisy patterns. This filter is given as $G(x, y)=1 /(\sqrt{2 \pi} \sigma) \exp \left[-\left(x^{2}+y^{2}\right) /\left(2 \sigma^{2}\right)\right]$, where $\sigma$ is the dispersion and $\sigma=1.5$ [a.u.]. For the computation of electric current density, we calculate the first term of Eq. (6) directly. The second term was shown to be negligible for the computation based on quantum mechanics [39].

In this paper, the atomic unit is adopted except for the case we explicitly state unit.

\section{RESULTS AND DISCUSSION}

\section{A. Non-conductive state}

In this subsection, aluminum nanowire models without electric current are studied by quantities based on Rigged QED. First, we study the pristine aluminum nanowire model shown in Fig. 1. In Fig. 4, the largest eigenvalue and its eigenvector of stress tensor density of the nanowire model are shown. The value of the largest eigenvalue of stress tensor is negative (positive) in blue (red) regions. The black solid line shows the nanowire surface where the 


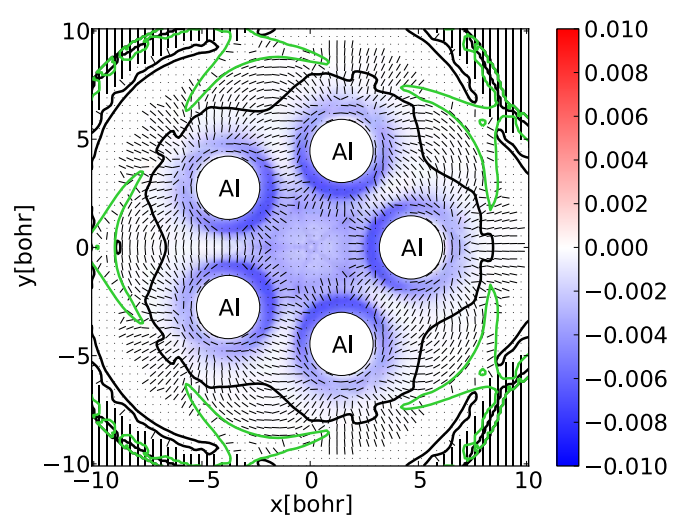

(a) $z=0$

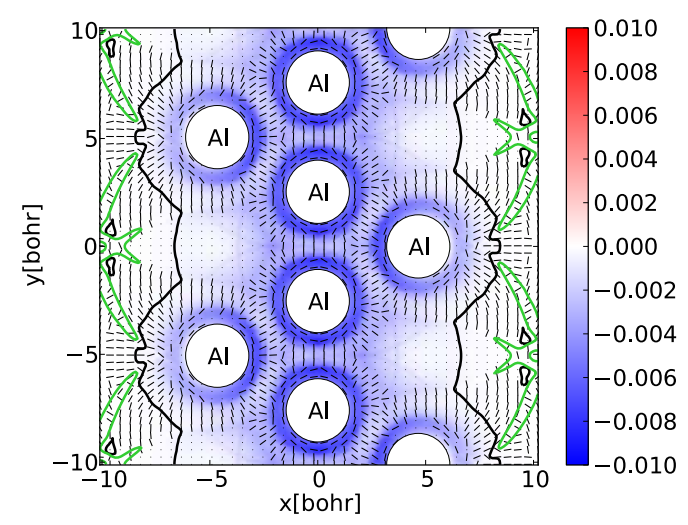

(b) $y=0$

FIG. 4: The largest eigenvalue and its eigenvector of the stress tensor density of the pristine aluminum nanowire model.

kinetic energy density is zero. The green solid line means that the eigenvalue is zero. All regions around aluminum nuclei are negative, and hence the value of the largest eigenvalue of the stress tensor density is negative in our aluminum nanowire model. Eigenvectors between aluminum nuclei are lined along the internuclear axis, where this distribution is called pseudo-spindle structure [40]. The pseudo-spindle structure is seen for all bonding between aluminum atoms. It is reported that metallic bond is distinguished from covalent bond by two features [31]. One is negative values of all eigenvalues of the stress tensor density, and the other is the degeneracy of eigenvalues of the stress tensor density. In our results, the former feature is clearly seen, since the largest eigenvalue is negative and the other eigenvalues are inevitably negative. The degeneracy cannot be judged only in Fig. 4, and the degeneracy on the internuclear axis between aluminum atoms is shown in Fig. 5. Panels (a), (b), and (c) are the results on the internuclear axes between aluminum atoms on a pentagon layer, between aluminum atoms on the atomic chain, and between aluminum atoms on a pentagon and the chain, respectively. From Fig. 5, it is seen that all Al-Al bonds have very similar distribution pattern of eigenvalues and shows clear degeneracy, where the difference between eigenvalues is within 0.005 [a.u.] around midpoints. Although the degeneracy should be judged at the Lagrange point, we have not checked where Lagrange point exists, since the current version of QEDynamics cannot calculate the Lagrange point for the output of OpenMX. However, Lagrange point certainly exists around the midpoint if exist, and hence we can know that the difference between eigenvalues is about 0.005 [a.u.]. Therefore, 


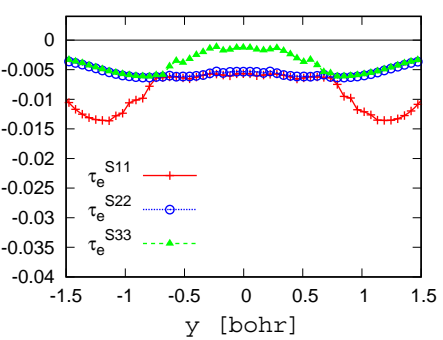

(a)

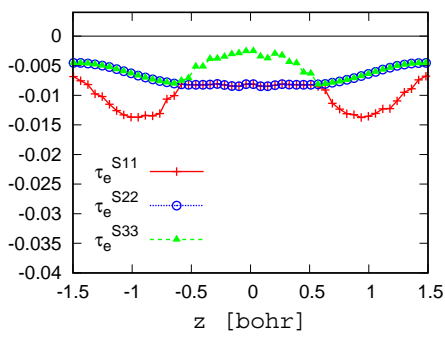

(b)

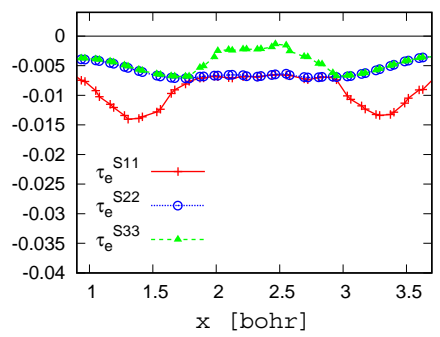

(c)

FIG. 5: The eigenvalues along internuclear axes in the aluminum nanowire. Panel (a) is the result on the internuclear axis between aluminum atoms on a pentagon layer. Panel (b) is that between aluminum atoms on the atomic chain. Panel (c) is that between aluminum atoms on a pentagon and the chain, where the result is shown as a function of $x$-coordinates. In all panels, the cross symbol with a solid line represents the first eigenvalue, the open circle with a dotted line represents the second eigenvalue, and the filled triangle with a dashed line represents the largest eigenvalue,

$\mathrm{Al}-\mathrm{Al}$ bonds in our nanowire model are confirmed to be metallic. Compared to typical metallic bond in lithium clusters [31], degeneracy is weaker, since the differences in lithium clusters are within 0.0005 [a.u.]. In the work, even $\mathrm{Li}_{2}$ shows stronger degeneracy, about 0.0002 [a.u.], and hence this stronger directionality in Al-Al bonds cannot be attributed to the structure of our model. We speculate that this difference of degeneracy arises from the difference between lithium and aluminum, since aluminum is considered to be metalloid occasionally. This confirmation of metallicity of aluminum nanowire supports that this nanowire model conducts electric current.

In comparison with the work [18], the shape of the surface with zero kinetic energy density is similar and the distribution pattern of the eigenvectors of stress tensor is also similar. However, the value of the largest eigenvalue of the stress tensor is larger than the work by about one order of magnitude. The result of the present paper is confirmed to be correct by the check of the data used in the previous work [18]. In regions outside of aluminum nanowire, arc-shape stripes are seen, and this pattern was also seen in the previous work. However, the distribution pattern in Fig. 4(b) is significantly different from the work [18]. This is attributed to the symmetry in the previous work. In the previous work, only reflection symmetry is realized in the computation and the result did not keep five-fold rotational symmetry. Hence, results are heavily dependent on which cross section is chosen. On the other hand, this symmetry is clearly realized in the present work. 


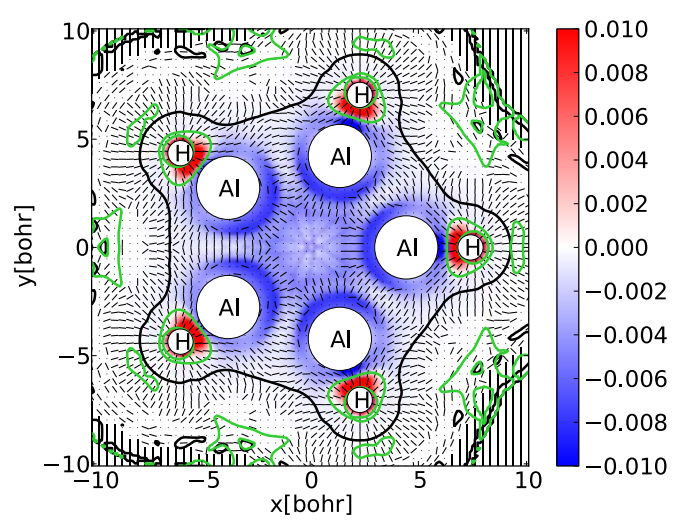

(a) $z=0$

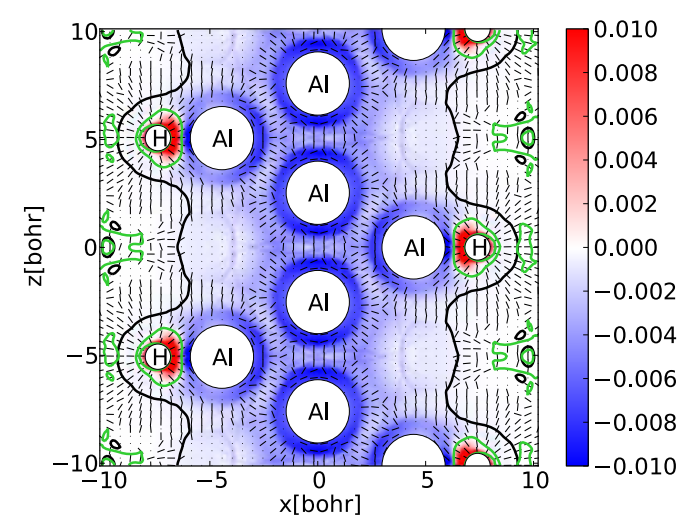

(b) $y=0$

FIG. 6: The largest eigenvalue and its eigenvector of the stress tensor density of hydrogen adsorbed aluminum nanowire model.

Next, hydrogen adsorbed aluminum nanowire model shown in Fig. 2 is investigated. The largest eigenvalue, $\tau_{e}^{S 33}$, and its eigenvector of stress tensor density of this nanowire model is shown in Fig. 6. The color map and lines are the same in Fig. 4. The largest eigenvalue and eigenvector of the stress tensor density hardly changes around aluminum nuclei. The bonding between aluminum and hydrogen atoms is classified as a covalent bond, since the spindle structure is formed. The region with positive values of $\tau_{e}^{S 33}$ exists between aluminum and hydrogen atoms and the distribution of eigenvectors connects two nuclei.

The degeneracy on the internuclear axis between atoms is shown in Fig. 7 for the clarification of the effect of hydrogen adsorption on the bonding between aluminum atoms. Panels (a), (b), (c), and (d) are the results on the internuclear axes between aluminum atoms on a pentagon layer, between aluminum atoms on the atomic chain, between aluminum atoms on a pentagon and the chain, and between an aluminum atom on a pentagon and the adsorbed hydrogen atom, respectively. It is seen that all $\mathrm{Al}-\mathrm{Al}$ bonds are not affected by the adsorption of hydrogen atoms, from the comparison between panels (a)-(c) of Figs. 5 and 7. Hence, this nanowire model remains conductor after the hydrogen adsorption. We can see that the covalent bond between $\mathrm{Al}$ and $\mathrm{H}$ atoms has strong directionality, since $\tau_{e}^{S 33}$ is positive for $x=6.4-6.9$ [bohr] and much larger than other two eigenvalues, in Fig. $7(\mathrm{~d})$. 


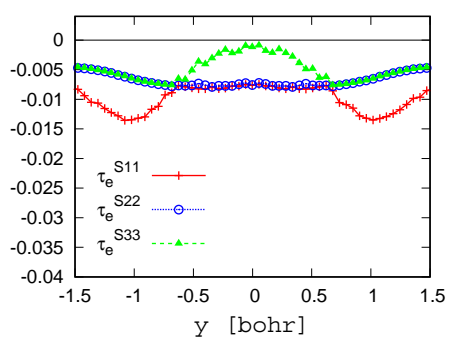

(a)

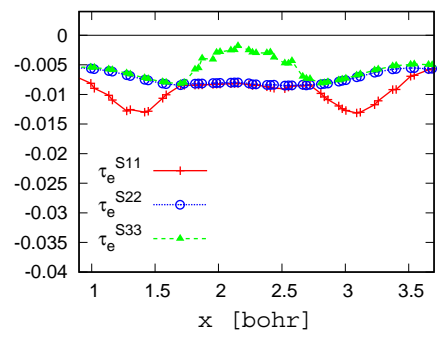

(c)

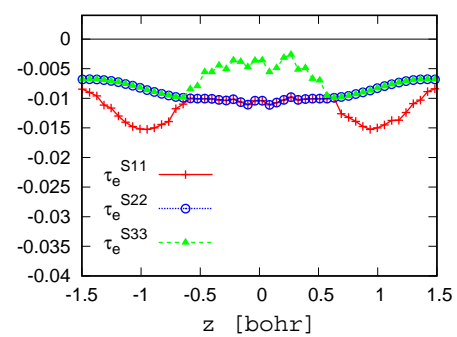

(b)

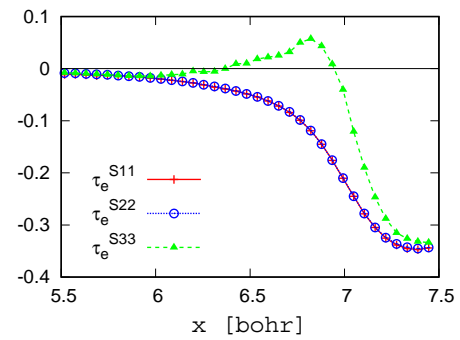

(d)

FIG. 7: The eigenvalues along internuclear axes in the aluminum nanowire. Panel (a) is the result on the internuclear axis between aluminum atoms on a pentagon layer. Panel (b) is that between aluminum atoms on the atomic chain. Panel (c) is that between aluminum atoms on a pentagon and the chain, where the result is shown as a function of $x$-coordinates. Panel $(\mathrm{d})$ is that between an aluminum atom on a pentagon and the adsorbed hydrogen atom. In all panels, the cross symbol with a solid line represents the first eigenvalue, the open circle with a dotted line represents the second eigenvalue, and the filled triangle with a dashed line represents the largest eigenvalue,

\section{B. Conductive state}

In this subsection, the effect of electric conduction on chemical bond in aluminum nanowire is studied by comparing with results shown in the previous section. First, the distributions of electric current for bias voltage at $0.3 \mathrm{~V}$ and $0.5 \mathrm{~V}$ are shown in Fig. 8. The results in the $y=0$ plane are shown and panels (a) and (b) are the results of $0.3 \mathrm{~V}$ and 0.5 $\mathrm{V}$, respectively. Electric current flows for the negative $z$-direction almost everywhere, and the value of the current is large around the aluminum chain. Electric current is seen to be slightly larger on the layer of pentagon than other layers.

The difference in the stress tensor density is studied for the purpose of the clarification of the change of chemical bonds. The change of the largest eigenvalue of stress tensor is shown in Fig. 9. The differences of $\tau_{e}^{S 33}$ at bias voltage $0.3 \mathrm{~V}$ and $0.5 \mathrm{~V}$ from $0 \mathrm{~V}$ are shown from 


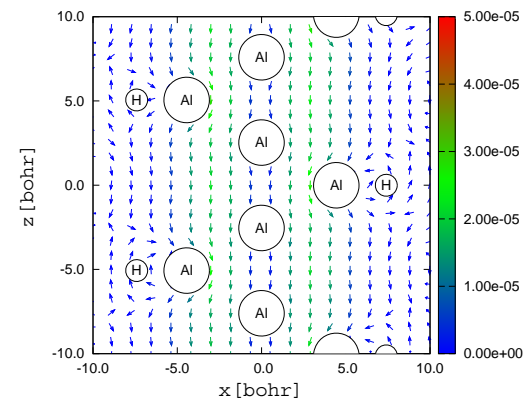

(a) $V_{B}=0.3 \mathrm{~V}$

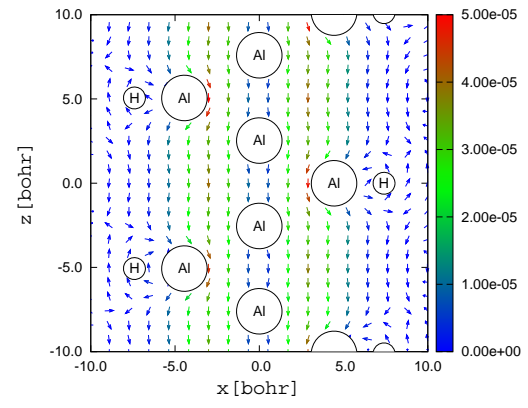

(b) $V_{B}=0.5 \mathrm{~V}$

FIG. 8: The distributions of electric current for bias voltage at $0.3 \mathrm{~V}$ and $0.5 \mathrm{~V}$. The results are shown in the plane, $y=0$. Panels (a) and (b) are the results of $0.3 \mathrm{~V}$ and $0.5 \mathrm{~V}$, respectively.

top to bottom, respectively. Panels (a) and (c) are the results on the $z=0$ plane, and panels (b) and (d) are those on the $y=0$ plane. The difference of $\tau_{e}^{S 33}$ in this figure is the largest eigenvalue and its eigenvector derived by diagonalizing the matrix of the difference between $\left.\stackrel{\leftrightarrow}{\tau}_{e}^{S}\right|_{V=V_{B}}$ and $\left.\stackrel{\leftrightarrow}{\tau}_{e}^{S}\right|_{V=0}$. Black solid lines show the surface that the difference of kinetic energy density is zero, $\Delta n_{T_{e}}=0$, and green solid lines mean no difference of the largest eigenvalue of stress tensor, $\Delta \tau_{e}^{S 33}=0$. The change of stress tensor is much smaller than the value of $\tau_{e}^{S 33}$ itself. The difference is large for larger bias voltage, while it is less than $10 \%$ even at $V_{B}=0.5 \mathrm{~V}$. This difference is positive in almost all region. This is because nanowire is destabilized with electric conduction and the stress tensor is related to the energy density as shown in Eq. (5). The difference of eigenvalues of stress tensor on internuclear axes between atoms is shown in Fig. 10 for the clarification of the change of stress tensor in regions of chemical bonds. Thick (thin) lines are results at $V_{B}=0.5(0.3) \mathrm{V}$. Both differences are much smaller than eigenvalues themselves for all bondings by the comparison between Figs. 7 and 10. Therefore, it is speculated that the effect of electric conduction on chemical bonding is small within $V_{B} \leq 0.5 \mathrm{~V}$.

In the previous work [25], the largest eigenvalue of stress tensor of an aluminum cluster was significantly changed by electric conduction. In their computations, they use the code computing electronic structure with electric current reported in Ref. [39]. In this code, effects by connection with electrodes are not included and electric current is implemented by unique procedure. First, its electronic structure computation is performed with the linear combination of atomic orbitals method, and its highest occupied 


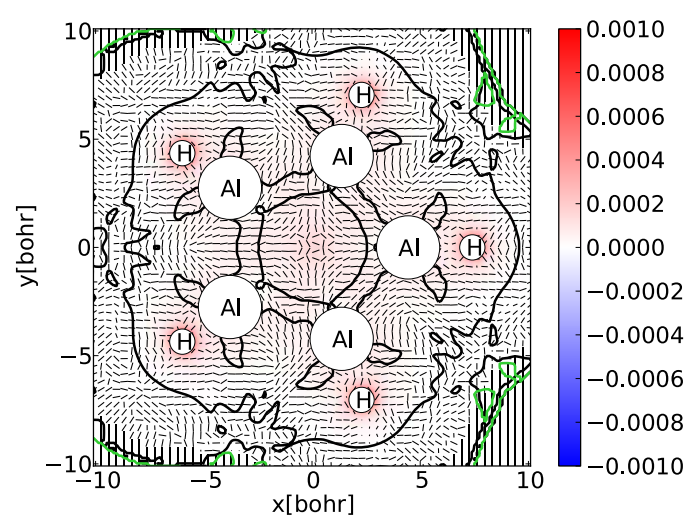

(a) $z=0$

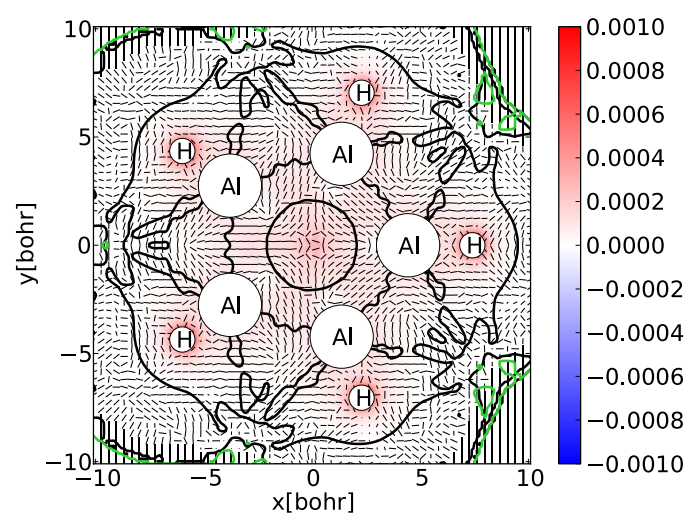

(c) $z=0$

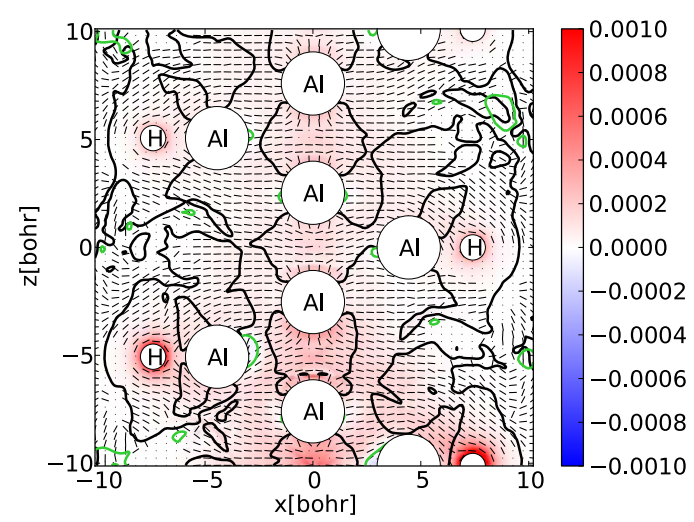

(b) $y=0$

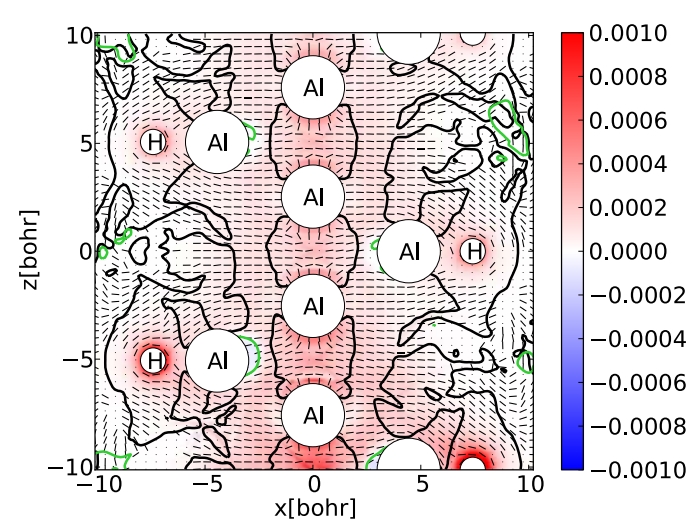

(d) $y=0$

FIG. 9: The difference of stress tensor for bias voltage at $0.3 \mathrm{~V}$ (panels (a) and (b)) and $0.5 \mathrm{~V}$ (panels (c) and (d)). Panels (a) and (c) are the results on the $z=0$ plane, and Panels (b) and (d) are those on the $y=0$ plane. Black solid lines show the surface of the difference of kinetic energy density is zero, $\Delta n_{T_{e}}=0$, and green solid lines mean the difference of the largest eigenvalue of stress tensor, $\Delta \tau_{e}^{S 33}=0$.

molecular orbital is forced to be mixed with a plane wave. This plane wave is expanded with Gaussian functions and is introduced for the purpose of the representation of ballistic conduction. This computational method may give much larger electric current than that by the NEGF method, and larger current should accompany more strong modification of electronic structure. Actually, the current density of single chain aluminum nanowire reported in Ref. [39] is much larger than our current density in Fig. 8 by about two orders of magnitude. Such a large current cannot be treated in the NEGF method. Hence, we cannot judge whether our results are consistent with their previous paper [25], 


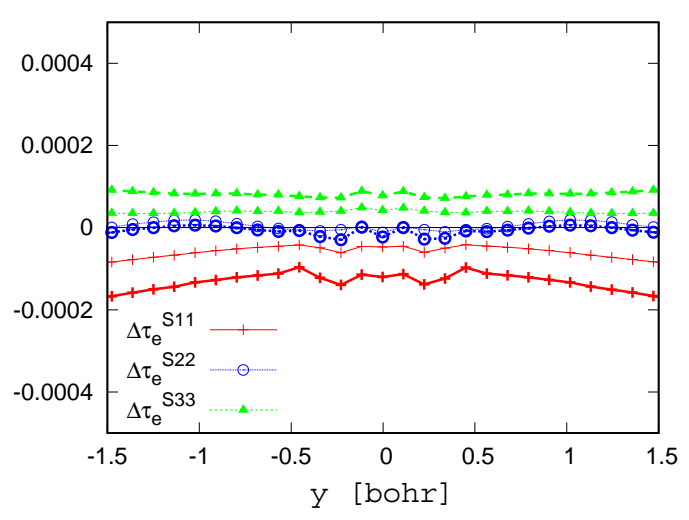

(a)

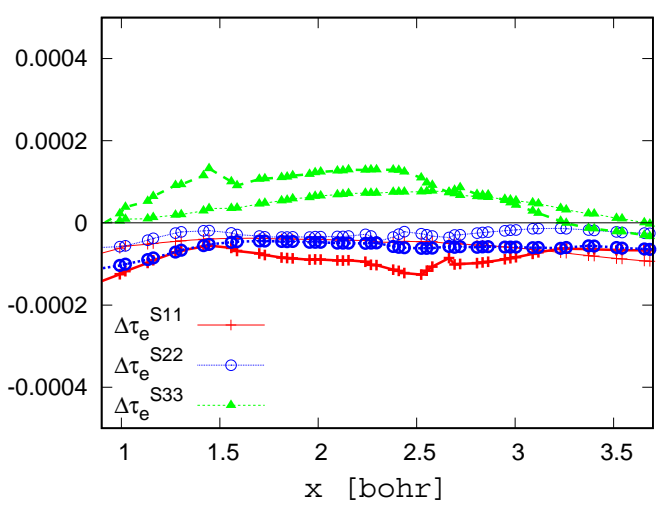

(c)

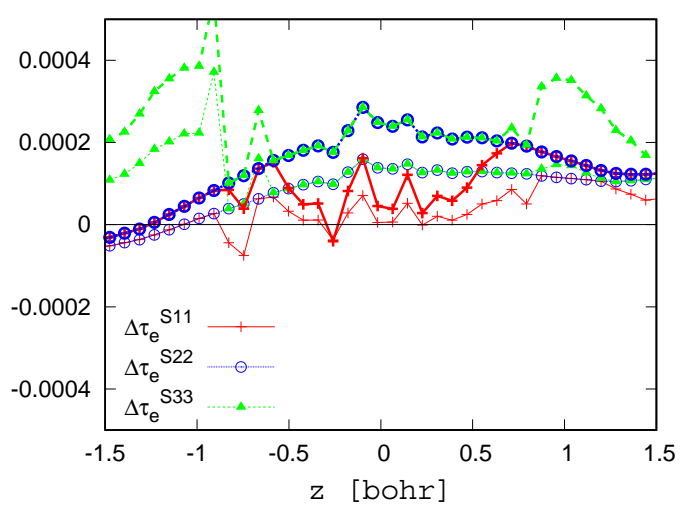

(b)

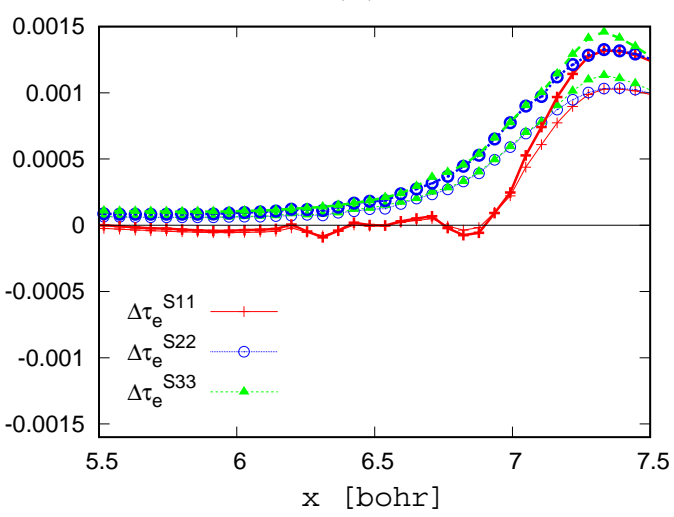

(d)

FIG. 10: The difference of eigenvalues of stress tensor on internuclear axes between atoms. The difference between results at $0 \mathrm{~V}$ and $0.5(0.3) \mathrm{V}$ is shown as thick (thin) lines. Panel (a) is the result on the internuclear axis between aluminum atoms on a pentagon layer. Panel (b) is that between aluminum atoms on the atomic chain. Panel (c) is that between aluminum atoms on a pentagon and the chain, where the result is shown as a function of $x$-coordinates. Panel (d) is that between an aluminum atom on a pentagon and the adsorbed hydrogen atom.

since we cannot directly compare the results. It is speculated that chemical bonding are inevitably affected by electric current if the current is large enough. We have confirmed that the current density about $10^{-4}$ [a.u.] is not enough to change chemical bonding significantly.

The bond order of chemical bonding is studied for the sake of the quantitative parametrization of the change of the chemical bonding. The bond order is defined with the energy density as seen from Eq. (4), and hence the difference of the energy density is shown in Fig. 11. The difference is calculated as the ratio of the energy density with a bias voltage to that without it, $\epsilon_{\tau_{\mathrm{V}}}^{S} / \epsilon_{\tau_{0}}^{S}$, on an internuclear axis between hydrogen and aluminum atoms. As mentioned above, we have not calculated where the Lagrange point exists. The 


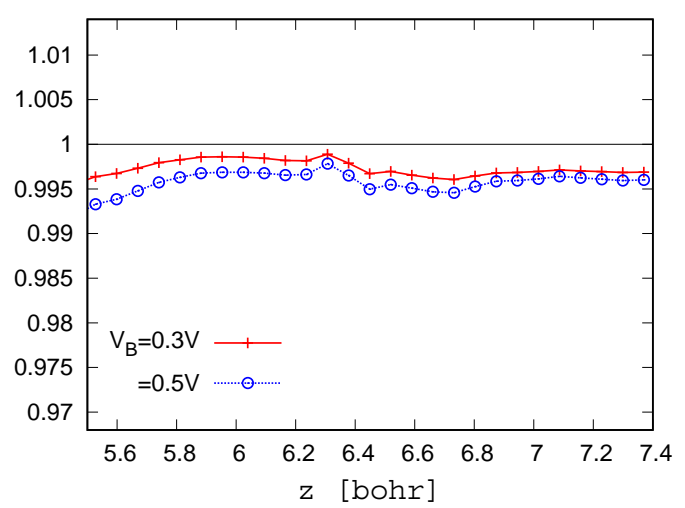

FIG. 11: The difference of the energy density on an internuclear axis between hydrogen and aluminum atoms is shown as a ratio of the result with bias voltages to that at $0 \mathrm{~V}$. The red line with cross marks is the result at $0.3 \mathrm{~V}$, while the blue line with open circle is that at $0.5 \mathrm{~V}$.

position of the Lagrange point is not changed significantly as seen from Ref. [32]. Hence, the Lagrange point exists probably a point closer to the hydrogen nucleus on the internuclear axis than the midpoint. From this figure, it is seen that the energy density, that is the bond order, is smaller for larger bias voltage. This confirms that the chemical bonding of the hydrogen atom on an aluminum atom is weakened for lager bias voltage, though the amount of the variation is less than $1 \%$ for $V=0.5 \mathrm{~V}$.

\section{SUMMARY}

In this paper, we have studied the effect of electric conduction on the chemical bonding between aluminum and hydrogen atoms in aluminum nanowire by the computation based on the NEGF method. First, it has been checked that our aluminum nanowire model and hydrogen adsorbed one have a conductive ability, since aluminum-aluminum bonding in them are metallic one. Then we have confirmed that the electric conduction weakens slightly the chemical bonding of hydrogen atom on aluminum nanowire and the amount of the variation is larger for larger bias voltage. However, the effect of the electric current on the chemical bonding is negligible for the bias voltage less than $0.5 \mathrm{~V}$. 


\section{Acknowledgments}

This work was supported by a Grant-in-Aid for Scientific Research (C) (17K04982) and Grant for Basic Science Research Projects from The Sumitomo Foundation. The computations were performed in part using Research Center for Computational Science, Okazaki, Japan. The authors also acknowledge Dr. Fukushima for the check of the result of our previous paper.

[1] J. Akola, H. Häkkinen, M. Manninen, Phys. Rev. B 58, 3601 (1998).

[2] V. Kumar, V. Sundararajan, Phys. Rev. B 57, 4939 (1998).

[3] J. Akola, M. Manninen, H. Häkkinen, U. Landman, X. Li, L.-S. Wang, Phys. Rev. B 60, 11297 (1999).

[4] M. Calleja, C. Rey, M. M. G. Alemany, L. J. Gallego, P. Ordejón, D. Sánchez-Portal, E. Artacho, J. M. Soler, Phys. Rev. B 60, 2020 (1999).

[5] B. K. Rao, P. Jena, J. Chem. Phys. 111, 1890 (1999).

[6] R. Ahlrichs, S. D. Elliot, Phys. Chem. Chem. Phys. 1, 13 (1999).

[7] O. Dolgounitcheva, V. G. Zakrzewski, J. V. Ortiz, J. Chem. Phys. 111, 10762 (1999)

[8] V. Kumar, S. Bhattacharjee, Y. Kawazoe, Phys. Rev. B 61, 8541 (2000).

[9] Q. Sun, Q. Wang, J. Z. Yu, V. Kumar, Y. Kawazoe, Phys. Rev. B 63, 193408 (2001).

[10] O. P. Charkin, D. O. Charkin, N. M. Klimenko, A. M. Mebel, Chem. Phys. Lett. 365, 494 (2002).

[11] Y. Kawakami, T. Kikura, K. Doi, K. Nakamura, A. Tachibana, Mater. Sci. Forum 426-432, 2399 (2003).

[12] O. P. Charkin, N. M. Klimenko, D. O. Charkin, A. M. Mebel, J. Russ, Inorg. Chem. 49, 1382 (2004).

[13] O. P. Charkin, N. M. Klimenko, D. O. Charkin, A. M. Mebel, J. Russ, Inorg. Chem. 49, 1898 (2004).

[14] O. P. Charkin, N. M. Klimenko, D. O. Charkin, J. Russ. Inorg. Chem. 50 S17, (2005).

[15] T. Makita, K. Doi, K. Nakamura, A. Tachibana, J. Chem. Phys. 119, 538 (2003).

[16] R.-H. Xie, G. W. Bryant, J. Zhao, T. Kar, V. H. Smith, Phys. Rev. B 71125422 (2005). 
[17] A. Goldberg and I. Yarovsky, Phys. Rev. B 75, 195403 (2007).

[18] A. Fukushima, K. Doi, M. Senami, and A. Tachibana, J. Power Sources 184, 60 (2008).

[19] D. J. Henry, A. Varano, I. Yarovsky, J. Phys. Chem. A 112, 9835 (2008).

[20] D. J. Henry, I. Yarovsky, J. Phys. Chem. A 113, 2565 (2009).

[21] D. J. Henry, A. Varano, I. Yarovsky, J. Phys. Chem. A 1135832 (2009).

[22] A. Varano, D. J. Henry, I. Yarovsky, J. Phys. Chem. A 114, 3602 (2010).

[23] A. Fukushima, K. Hirai, M. Senami, A. Tachibana, Surf. Sci. 604, 1718 (2010).

[24] D. J. Henry, P. Szarek, K. Hirai, K. Ichikawa, A. Tachibana, I. Yarovsky, J. Phys. Chem. C 115, 1714 (2011).

[25] K. Ichikawa, Y. Ikeda, R. Terashima, A. Tachibana, Mater. Sci. Forum, Vols. 706-709, 1539 (2012).

[26] For example, see, A. Tachibana, New Aspects of Quantum Electrodynamics, Springer (2017).

[27] A. Tachibana, In Fundamental World of Quantum Chemistry, A Tribute to the Memory of Per-Olov Löwdin; E. J. Brändas, E. S. Kryachko, Eds, Kluwer Academic, Dordrecht, 2003, Vol. 2, p. 211.

[28] A. Tachibana, J. Mol. Model, 11, 301 (2005).

[29] A. Tachibana, J. Mol. Struct. (THEOCHEM) 943, 138 (2010).

[30] P. Szarek and A. Tachibana, J. Mol. Model. 13, 651 (2007).

[31] H. Nozaki, Y. Ikeda, K. Ichikawa, A. Tachibana, J. Comput. Chem. 36, 1240 (2015).

[32] H. Nozaki, M. Senami, K. Ichikawa, A. Tachibana, Jpn. J. Apl. Phys. 55, 08PE01 (2016).

[33] A. Tachibana, J. Chem. Phys. 115, 3497 (2001).

[34] H. Nozaki, Y. Fujii, K. Ichikawa, T. Watanabe, Y. Aihara, A. Tachibana, J. Comput. Chem. 37,1924 (2016).

[35] P. Szarek, Y. Sueda, and A. Tachibana, J. Chem. Phys. 129, 94102 (2008).

[36] T. Ozaki et al., OpenMX package, http://www.openmx-square.org/.

[37] T. Ozaki, Phys. Rev. B 67,155108 (2003).

[38] QEDynamics, M. Senami, K. Ichikawa, A. Tachibana https://github.com/mfukudaQED/QEDalpha.

[39] M. Senami, Y. Ikeda, A. Fukushima, A. Tachibana, Jpn. J. Appl. Phys. 49, 115002 (2010).

[40] K. Ichikawa, A. Wagatsuma, P. Szarek, C. Zhou, H. Cheng and A. Tachibana, Theor. Chem. Acc. 130, 531 (2011). 